\title{
EL ROL DEL ESPACIO RECREADO EN LA CONSTRUCCIÓN DISCURSIVA DE IDENTIDAD DE ADOLESCENTES MAPUCHES DE TEMUCO Y SANTIAGO ${ }^{1}$
}

The role of space as recreated in the discourse identity construction of Mapuche adolescents from Teтuco and Santiago

\author{
Héctor Turra* \\ Sandra Garrido \\ Chery Pérez \\ Gabriel Llanquinao \\ María Eugenia Merino
}

Resumen

El presente artículo describe la conexión entre los espacios culturales rurales de la cultura mapuche y aquellos recreados en centros urbanos y su vinculación con la construcción de la identidad étnica en adolescentes mapuches urbanos. El estudio es cualitativo descriptivo y los datos se abordan desde el enfoque de análisis del discurso de la psicología discursiva (Edwards y Potter, 1998, 2000) y el enfoque de identidades espaciales de Proshansky (1978). La muestra corresponde a treinta adolescentes mapuches hombres y mujeres residentes en la ciudad de Santiago y treinta de la ciudad de Temuco, quienes participaron en entrevistas conducidas por un entrevistador mapuche. Los resultados revelan que el rol de los espacios culturales recreados en contextos urbanos es desarrollar una función mediadora del cambio (Proshansky et al., 1983) desde el lugar de origen a nuevos espacios lo que permite reforzar la identidad étnica de los adolescentes.

Palabras clave: Espacios recreados, Identidad de lugar, Identidad mapuche, Función mediadora del cambio, Análisis del discurso.

\section{Abstract}

This article describes the connection between Mapuche rural cultural spaces and those re-created in urban areas and their connection with the construction of ethnic identity in urban Mapuche adolescents. This is a qualitative and descriptive study and the data is analyzed through the approach of discourse analysis of discursive psychology (Edwards y Potter, 1998, 2000) and considers the notion of place identity by Proshansky (1978). The research population were thirty Mapuche adolescents living in Santiago and thirty living in Temuco. These adolescents were interviewed by a Mapuche interviewer. The findings reveal that the re-created urban cultural spaces fulfill a mediating role in the change from original places to new ones (Proshansky et al., 1983), which allows these adolescents to reinforce their ethnic identity construction.

\footnotetext{
${ }^{1}$ Este trabajo da cuenta de uno de los objetivos del proyecto Fondecyt 1090523 "Construcción discursiva de la identidad étnica de adolescentes mapuches de Temuco y Santiago", que devela el rol del espacio y lugar en la construcción de la identidad étnica en los jóvenes mapuches urbanos.
} 
Key words: Recreated spaces, Place-identity, Mapuche identity, Mediating role, Discourse analysis.

\section{INTRODUCCIÓN}

En Chile la identidad mapuche ha sido abordada desde variadas perspectivas y disciplinas en las últimas décadas. Por ejemplo Tripailaf (1969) desde una perspectiva educacional plantea que las relaciones que se dan entre la cultura mayoritaria y minoritaria se encuentran marcadas por la discriminación racial generando un complejo de inferioridad en los estudiantes mapuches de distintos niveles educacionales. Del mismo modo Aillahuil (1996) caracteriza la relación entre personas del pueblo mapuche y chilenas como basadas en la discriminación y enfatiza la influencia de la escuela sobre la construcción de identidad del niño mapuche y como dicha entidad promueve la discriminación. Por otro lado, Bello (2002) describe cómo la migración mapuche a la ciudad de Santiago configura y resignifica la identidad en el ámbito urbano, y plantea que los mapuches sustentan su propia identidad basada en recuerdos y en la recreación de prácticas culturales realizadas en un nuevo contexto. Asimismo elaboran sus propias concepciones de identidad como son pertenecer a un pueblo con historia inmemorial sustentado en el linaje o kupan, en prácticas, creencias, religiosidad y lugar de origen, todo lo cual constituye un tronco común que los une y los determina como mapuches, de forma individual y colectiva.

Lo planteado, Bello cobra singular relevancia para el presente estudio, ya que se identifica al lugar como un referente que incide y determina la construcción de identidad étnica. Ello debido a que las diferentes culturas que habitan en un mismo territorio geográfico generan espacios propios en donde practican rituales y se celebran tradiciones que colaboran en la preservación de la identidad étnica, y asimismo se constituyen en ejes de afiliación cultural. Es el caso de las personas mapuches que se identifican y se reconocen como parte de dicho grupo, y han manifestado a lo largo de la historia de la nación chilena diversos modos de identificación, pertenencia y apego a los espacios ancestrales mediante, que ha incidido en un compromiso de preocupación permanente por la preservación de los lugares que pertenecieron históricamente a su pueblo. Ello se constituye en un dilema en especial para los mapuches que han emigrado a las urbes sobre todo por razones económicas desde sus comunidades de origen. Se trata de un conflicto en torno a la propia identidad que incluso puede detonar efectos psicológicos y sociales que afecten en forma negativa, o en algunos casos culminen incluso reforzando su identificación étnica (Merino, Mellor, Saiz y Quilaqueo, 2009). En consecuencia, al residir en espacios urbanos alejados de sus comunidades de origen los mapuches migrados desarrollan la necesidad de "sentirse en casa" y buscan en consecuencia "recrear" dichos espacios. 
Esto constituye el problema del presente estudio. Nos interesa conocer el rol de los espacios 'recreados' en ámbitos urbanos en la construcción discursiva de identidad étnica de adolescentes mapuches, quienes han emigrado o cuyas familias hayan emigrado de sus lugares originales y que ahora residen en las ciudades de Temuco y Santiago. Para ello se identifican los espacios recreados por los jóvenes de ambas ciudades y se caracteriza la relación que los adolescentes atribuyen hacia dichos lugares, lo que permite describir el rol y las funciones que estos cumplen en la construcción de su identidad étnica. Para abordar ambos objetivos se analiza el discurso oral de sesenta jóvenes mapuches de Temuco y Santiago mediante dos enfoques: la psicología discursiva de Edwards y Potter $(1998,2000)$ y de identidades espaciales de Proshansky (1978).

\section{MARCO TEÓRICO}

Identidad, lugar y discurso

El lugar no solo es un espacio donde habitar, sino que representa a la persona en sí misma, debido a que el lugar se relaciona y representa a la propia familia, las actividades y las prácticas de la persona y/o de la cultura a la que pertenece (Proshansky et al., 1983). En este contexto la identidad es entendida como un proceso de cambio continuo (Wetherell, 2009) y es definida y delimitada por varios factores entre los cuales se encuentran los lugares físicos y sociales que forman parte de la biografía de las personas. Una de las disciplinas que han abordado las conexiones entre identidad y lugar es la psicología ambiental. Heimstra y McFarling (1979) definen la psicología ambiental como la disciplina que se enfoca en el conjunto de relaciones generadas entre el medioambiente físico de las personas y su comportamiento. Esta disciplina, perteneciente al enfoque cognitivista, fue la primera en establecer dicha relación. Sin embargo, desde varias otras perspectivas el lugar ha sido estudiado como uno de los aspectos que inciden en la construcción de identidad (Proshansky, Fabian y Kaminoff, 1983; Twigger-Ross y Uzzell, 1996; Lalli, 1988; Valera y Pol, 1994; Altman y Low, 1992; Hidalgo, 1998; Knez, 2005; Valera, 1996; McAndrew, 1998). Estos autores han profundizado en dicha relación relevando aspectos cognitivos, sociales y emocionales.

El concepto de identidad de lugar es definido por Proshansky, Fabian y Kaminoff, como una subestructura de la identidad del ser:

(...) es una subestructura de la identidad del ser de un individuo consistente en cogniciones ampliamente concebidas del mundo físico en el que viven los individuos. Estas cogniciones representan recuerdos, ideas, sentimientos, actitudes, valores, preferencias, significados y concepciones del comportamiento y experiencias que están relacionadas con los variados y complejos escenarios físicos que definen la existencia del día a día de 
todo ser humano. En el centro de tales cogniciones está el 'medioambiente pasado' de la persona; un pasado que consiste de lugares y espacios y de sus propiedades que han servido de forma instrumental en la satisfacción de necesidades biológicas, psicológicas, sociales y culturales (1983:59) (traducción propia).

Como se observa, la identidad de lugar posee componentes netamente cognitivos, sin embargo, para el propósito del presente estudio se opta por un enfoque más social en torno a la construcción de identidad y su relación con los espacios. Así, esta conexión estará determinada por la forma cómo los adolescentes mapuches de Temuco y Santiago expresan el vínculo identidad-lugar en su discurso oral. Desde esta perspectiva, la identidad se constituye para estos jóvenes en un constructo cambiante que evoluciona y se adapta a diferentes contextos y situaciones. Es además de carácter dilemático y se genera discursivamente mediante afiliaciones temporales y relacionales, que son evidenciadas por la utilización de categorías culturales y repertorios específicos (Hall, 2006).

\section{LA IDENTIDAD ÉTNICA Y LOS ESPACIOS SIMBÓLICOS}

Para comprender la identidad étnica es necesario comprender la visión que el "otro" tiene sobre la persona y el concepto de alteridad (otherness) para así establecer las diferencias entre el ser étnico y la cultura dominante (Merino y Tileaga, 2010:2). En este sentido pertenecer a un grupo minoritario facilita la construcción de identidad de sus miembros, ya que la alteridad de estos grupos constituye un encauce de los procesos de formación de identidad (Merino y Tileaga, 2011). En efecto, la formación de aquella es concebida como el resultado de un conjunto de procesos interrelacionados como los procesos cognitivos, evaluativos y de categorización (Tajfel y Turner, 1979). En este contexto las actividades sociales que se han llevado a cabo dentro de espacios y lugares delimitados gatillan procesos psicológicos referidos a estas dinámicas sociales, las que son reflejadas en el discurso (Edwards, 1997). El lugar no es un espacio vacío y sin significados para sus habitantes, por el contrario, el concepto involucra un conjunto de actividades y dinámicas sociales que inciden en la identidad de las personas (Dixon y Durrheim, 2000). Para la psicología ambientalista la noción de lugar y espacio se construye en la cognición de las personas, ya que las caracterizaciones geográficas, sociales, entre otras, ocurren allí (Dixon y Durrheim, 2000); sin embargo, Danziger (1997) puntualiza que la psicología se expresa en los espacios interpersonales de la conversación.

Por otra parte, al ser la identidad un constructo cambiante y dinámico construido y re-construido permanentemente, demuestra que se trata de un fenómeno que ocurre a nivel cognitivo y se ejecuta a nivel pragmático mediante 
acciones discursivas (Merino y Tileaga, 2010). No obstante, la construcción de identidad se ve determinada por las características particulares de un grupo minoritario, esta es constantemente redefinida por los individuos, y es influenciada por diversos repertorios pertenecientes a los distintos grupos sociales (Benwell y Stokoe, 2006). Al cambiar de lugar y establecer nuevas relaciones sociales, la identidad se adapta y se redefine, las identidades se constituyen en múltiples, dilemáticas y construyéndose por intermedio de repertorios discursivos producidos en espacios y tiempos determinados (Hall, 2001). En este sentido Wetherell (2009) plantea que la identidad étnica debe ser construida una y otra vez pues necesita nacer constantemente.

Cada cultura posee sus propios espacios simbólicos y tradicionales que las identifica y los distingue y con los cuales los miembros de un grupo se sienten identificados (Dixon y Durrheim, 2000). Para las culturas minoritarias estos espacios operan como diferenciadores o marcadores de alteridad en relación con las culturas dominantes. Los espacios simbólicos para las personas pueden variar desde una ciudad específica, o un país, la diferenciación entre 'persona de ciudad'/'persona de campo', a lugares más reducidos como el ngillatuwe (lugar donde se celebre el rito religioso —ngillatun - más relevante de la cultura mapuche). Estos lugares a los que las personas pertenecen y han pertenecido moldean las preferencias que poseen sobre los lugares (Hauge, 2007); es decir, los espacios biográficos de las personas determinan sus preferencias presentes y futuras en cuanto a los lugares que se escogen para establecer un nuevo hogar. Además, los lugares no poseen características per se, si bien son preferidos por los individuos por las características naturales que poseen, estos pueden ser modificados y personalizados para poder adaptarse y reflejar las particularidades identitarias del individuo (Hauge, 2007), y así lograr el sentimiento de 'sentirse en casa'.

\section{DILEMA DE LO URBANO Y LO RURAL}

El pueblo mapuche ha sido definido tradicionalmente como habitantes "del campo"; sin embargo, estadísticas recientes han demostrado que alrededor de un $70 \%$ de la población mapuche reside en urbes (Bello, 2002). La migración desde espacios rurales a urbanos está delimitado por diversos factores entre los cuales se encuentran razones económicas y de estudio, pero ¿cuál es el lugar donde realmente pertenecen y se identifican los mapuches?

El entorno urbano es considerado no solo un escenario físico, es también una dimensión simbólica donde se llevan a cabo interacciones sociales entre personas que comparten un entorno urbano (Castell, 2004, ed., 16). Sin embargo, la definición y diferenciación entre lo urbano y lo rural es compleja. Usualmente ambos conceptos están relacionados con un conjunto de criterios de delimitación, como por ejemplo la densidad de la población, características socioculturales, población que trabaja en el sector agropecuario o simplemente la ubicación geográfica (Barros, 2005). No 
obstante estos criterios puedan parecer objetivos, lo urbano y lo rural también contienen aspectos subjetivos que nacen desde la percepción de los individuos; ambos contextos son caracterizados por los individuos mediante categorías que reflejan la subjetividad que se les otorga a los espacios. Por ejemplo, se establece que las ciudades son un centro de desarrollo académico que ofrece mejores oportunidades para una mejor calidad de vida; por otro lado, se definen las áreas rurales como más seguras y tranquilas (Corbett, 2007).

El dilema entre lo urbano y lo rural está basado en estas y otras percepciones que manejan los individuos, quienes reconocen características positivas y negativas de ambos contextos. Es en este punto donde el concepto de "migración" juega un rol preponderante. De acuerdo con Papastergiadis (2000) en el contexto de la vida individual, la movilidad o migración es endémica al contexto mundial de globalización. Más aún, la movilidad desde espacios rurales hacia urbanos se da principalmente a través de la educación, ya que es esta la que promueve la automovilización tanto social como espacial (Corbett, 2005; 2007).

La población mapuche en Chile reside en la actualidad en ciudades, a pesar de la asociación histórica mapuche-rural que impera en la sociedad mayoritaria. Esta situación se puede explicar esencialmente debido a la "disminución y degradación de las tierras que pertenecen al pueblo mapuche, lo que genera la expulsión de sus lugares de origen" (Bello, 2002:2). La discusión sobre lo urbano y lo rural es relevante en el sentido que devela un aspecto importante de la identidad mapuche, la cual está fuertemente marcada por su relación con la tierra y con la naturaleza en general (Bello, 2002). Los denominados 'mapuches urbanos' es un concepto que ha sido utilizado por diversos autores (Bello, 2002; Aravena, 2003, Kropff, 2004, entre otros), y con frecuencia se refiere a aquellas personas que son hijos o nietos de mapuches que han emigrado a ciudades y que han perdido los vínculos con la naturaleza, tierra, tradiciones y costumbres (Bello, 2002). Sin embargo, no todos los mapuches urbanos han perdido sus tradiciones y costumbres, ya que algunas de estas se celebran en centros urbanos, como en Santiago y Temuco, en espacios especialmente diseñados, adaptados y recreados para estos propósitos.

\section{ESPACIOS RECREADOS: FUNCIÓN MEDIADORA DEL CAMBIO}

Al emigrar a lugares distintos a los de origen, los miembros de un grupo minoritario y/o indígena pueden experimentar diversos fenómenos asociados. Uno de ellos es la discriminación y/o segregación. Dixon y Durrheim (2004) plantean que estos fenómenos son factores preponderantes en la relación que se genera entre el ser y los espacios. La segregación experimentada por personas pertenecientes a grupos culturales minoritarios genera en ellas la necesidad de una reorganización del espacio y el lugar. Este fenómeno es entendido como una forma de "dislocación" (Dixon y Durrheim, 2004:1) que involucra diferentes 
formas de acomodación y de adaptación permitiendo que la transición desde lo rural a lo urbano sea menos abrupta.

Proshansky et al. (1983) explican que cuando una persona percibe discrepancias entre el escenario físico y su propia identidad, ejecuta ciertos procesos cognitivos que permiten su adaptación al lugar. Este fenómeno, denominado "función mediadora del cambio" (mediating change function), es sustentado primeramente por procesos cognitivos relacionados con la adaptación del nuevo lugar. El individuo adapta las condiciones físicas del nuevo lugar a sus necesidades basándose en aquellas cogniciones que lo remontan a lugares de su biografía (Hormuth, 2010). Un segundo tipo de cognición se refiere a aquellas basadas en el mundo físico de las transacciones interpersonales. Las interacciones son influenciadas por el lugar físico donde se llevan a cabo; y al mismo tiempo, las actividades que las personas desarrollan alteran la naturaleza física del espacio. En otras palabras, el individuo adapta los espacios para que allí se desarrollen las interacciones sociales que le interesan y aportan a su identidad. Según Hormuth (2010) las interacciones son determinadas por el lugar, y el significado de ese mismo espacio puede ser alterado por dichas interacciones. En definitiva, tanto la función mediadora del cambio como las transacciones interpersonales apuntan a adaptar los espacios como un medio para regular los procesos privados y tienen como propósito cambiar ya sea el espacio físico o el significado de este (Proshansky, et al., 1983). Más aún, para estos autores existe un tercer tipo de cognición: la "competencia ambiental" o modificación de la propia identidad. Se trata de la habilidad para actuar de forma apropiada en la relación que el ser tiene con un contexto físico. Cuando las características del lugar no permiten modificaciones y los significados de este no pueden ser modificados, se activa una cognición que apunta a cambiar a la persona en sí con el fin último de optimizar la relación persona-lugar. Este proceso entonces requiere de la modificación de aspectos cognitivos propios de la identidad de la persona, lo que permite generar una relación estable con el ambiente en que se habita. Ello puede implicar el cambio de ciertos input sensoriales para minimizar los efectos de algunos estímulos o de ciertas relaciones interpersonales determinadas (Hormuth, 2010). Desde esta perspectiva, los espacios recreados surgen como una respuesta a la incompatibilidad entre identidad y espacio físico, es decir, producto de su alteridad la persona desconoce y hasta cierto punto rechaza el nuevo espacio, lo que lo lleva a generar estrategias que permitan el ajuste entre ambos componentes. Un espacio recreado desarrolla una función mediadora de cambio y es una manifestación clara de la dislocación que se ha producido con el lugar actual donde una persona desarrolla su socialización con el mundo. 


\section{METODOLOGÍA}

\section{Diseño}

El presente estudio es cualitativo-descriptivo, asume el enfoque de "identidades de lugar" (place identity) propuesto por Proshansky, Fabian y Kaminoff (1983) y desarrolla su análisis considerando el enfoque discursivo de identidades espaciales de Dixon y Durrheim (2000). Es por ello que este artículo se centra en cómo los hablantes expresan discursivamente el rol de espacios tradicionales mapuches y cómo estos son recreados en centros urbanos.

\section{Participantes, procesos y materiales}

Sesenta adolescentes mapuches, hombres y mujeres que residen en las ciudades de Temuco y Santiago participaron en entrevistas tipo conversación sobre su identificación étnica. Este técnica permite generar un dialogo cómodo y fluido entre entrevistado y entrevistador sobre la base de una pregunta inicial ¿Qué significa para ti ser un mapuche en la sociedad chilena actual? Todas las entrevistas fueron conducidas por entrevistadores mapuches, lo que permitió que tanto entrevistador como entrevistado se comprometieran cooperativamente en "producir la entrevista", las que fueron audio-grabadas en espacios elegidos por los jóvenes.

\section{Método de análisis}

Para el análisis de la muestra se utilizó el análisis del discurso desde el enfoque de la psicología discursiva (Edwards, 1997; Edwards y Potter, 1998; Wetherell y Potter, 1992). Este enfoque, de carácter hipotético-deductivo, se sustenta en las "acciones discursivas" interpersonales que las personas llevan a cabo en distintos entornos culturales y que constituyen una vía para el entendimiento de las vidas de los individuos. Es decir, en nuestro estudio interesan las actividades situadas de habla tanto del entrevistado como del entrevistador quienes, en acciones discursivas conjuntas, construyen su discurso en torno a la identificación de ser mapuche en la sociedad chilena actual. Ello comprende acciones y posicionamientos discursivos en torno a los temas que se defienden y argumentan (stakes) respecto de la forma como cada hablante construye la relación entre mente y mundo, los scripts mentales y su disposición hacia la acción, la construcción de hechos, la categorización y membresía con determinadas categorías y la particularización de acciones y eventos (Edwards y Potter, 2000; Hepburn y Wiggins, 2007).

Por otra parte, para analizar la conexión entre los espacios físicos y sociales en la construcción de la identidad se utilizó el enfoque de identidad de lugar de Proshansky et al. (1983). Desde esta perspectiva se buscó relevar la función 
atribuida a estos espacios en el proceso de construcción de identidad étnica, y además establecer conexiones entre los fenómenos de dislocación (Dixon y Durrheim, 2000) y los espacios recreados para poder determinar la incidencia de la dislocación sobre la creación y participación en dichos espacios.

\section{ANÁLISIS Y DISCUSIÓN}

A continuación se describen tres categorías de lugar a las que los jóvenes aluden de manera recurrente y a las que atribuyen determinadas características. Se trata de la ciudad, los espacios culturales recreados, y la escuela y liceo.

\section{La ciudad}

La cultura mapuche tradicional se caracteriza por la relación que sus miembros establecen entre el hombre, la tierra y la naturaleza. Es por esta razón que es característico que los mapuches habiten en zonas rurales donde el contacto con la naturaleza es más directo y cercano. En la cosmovisión mapuche el vínculo existente entre la naturaleza y el hombre es considerado sagrado e inseparable uno del otro ( $a d m a p u)$, siendo esta relación la que permite la coexistencia entre ambas entidades. Sin embargo, a pesar de lo relevante de esta relación hombrenaturaleza, en las últimas décadas muchos miembros de esta cultura han emigrado desde áreas rurales a diferentes ciudades de Chile, principalmente a Santiago, la capital, y Temuco, capital de la región de La Araucanía. De acuerdo los jóvenes de Santiago este fenómeno migratorio se debe a diversos factores, como son mejores servicios, urbanización, campo laboral o de estudios, entre otros, tal como lo destaca Jo de Santiago ${ }^{2}$ :

"aquí en Santiago como hay más urbanización uno tiene que: el mayor campo laboral que hay es acá en Santiago, eso es lo que encuentro yo y que uno tiene que estudiar para poder tener una carrera de profesión y todo eso" S:H:Jo.

En el discurso de Jo se releva el concepto de "urbanización" y aunque no se encuentra explícitamente definido, se trata de una categoría que posee sus propias características y que es diferente a lo rural o al "campo". Jo le atribuye a la ciudad de Santiago ciertos atributos en tanto espacio donde existe un amplio "campo laboral ". Se trata de un lugar donde los adolescentes pueden desarrollarse y tener un mejor estilo de vida. Otra referencia a la ciudad de Santiago tiene que ver con las

\footnotetext{
${ }^{2}$ La sigla usada para la codificación de los extractos es la siguiente: primera letra corresponde a la ciudad de residencia del entrevistado T "Temuco" y S "Santiago". La segunda letra representa el sexo del adolescente H "hombre" M "mujer" y finalmente la última letra representa la inicial del nombre del entrevistado.
} 
oportunidades académicas lo que puede mejorar la calidad de vida y permite desarrollarse en varios ámbitos, también hacen mención a las remuneraciones, que son mejores en la ciudad que en el campo, y el estilo de vida citadino les permite adaptarse de mejor forma al mundo laboral. Para los jóvenes los centros urbanos ofrecen mayor variedad y oferta en general para poder estudiar y acceder a una profesión que les permite acceder a una mejor calidad de vida.

Los jóvenes de Temuco comparten dichos atributos a los que agregan que se trata de un espacio donde predominan apellidos mapuches, donde se realizan celebraciones y actividades mapuches en espacios culturales, y que es visitado por muchas personas mapuches provenientes de diferentes lugares de la región. Los atributos positivos que los adolescentes identifican tanto en Santiago como en Temuco muestran la presencia del componente afectivo evaluativo (Proshansky, 1978), ya que los jóvenes se identifican con un lugar al cual le otorgan determinadas características que permiten generar un ambiente emocional positivo.

Sin embargo, también se relevaron cualidades negativas atribuidas a la ciudad, aludiéndose, por ejemplo, a la dificultad de acceder al conocimiento sobre la cultura mapuche, en particular sobre el significado de sus apellidos, tal como lo describe Jo de Santiago: "pa' allá pa' Temuco que de allá generalmente viene la población indígena yo creo que allá es más fácil conseguir un conocimiento del apellido indígena, del apellido mapuche en este caso" S:H:Jo. También los adolescentes de Santiago atribuyen a esa ciudad rasgos negativos como el estilo de vida marcado por el apuro, enojo, estrés y contaminación, como se observa en el discurso de $\mathrm{P}$.

“(...) la verdad es que la ciudad me gusta por el hecho de que a lo mejor uno puede establecer mejores situaciones económicas todo eso, pero no por el hecho de quedarse porque acá la contaminación, la gente, la misma gente es la que aburre, la gente acá es otro estilo de vida la gente vive apurada, vive enojada, estresada" S:M:P.

Por su parte Temuco destaca como un espacio de conflictos por demandas territoriales y la presencia de una discriminación más abierta. A este respecto los jóvenes argumentan que al ser Temuco una ciudad más pequeña en términos de población y tamaño, y por su cercanía con las comunidades mapuches, la presencia indígena es más visible, lo que puede significar mayor discriminación. Es el caso de Cla quien desarrolla en su discurso el dilema entre "ser" y "sentirse" mapuche debido a los episodios de discriminación vividos; es decir, posicionarse como mapuche solo por el apellido o características físicas, o bien comprometerse activamente con la cultura, sus valores y prácticas culturales. Obsérvese este dilema desplegado mediante un argumento contradictorio entre las líneas 167 y 169 de la entrevista. 
[líneas 3 a 6 ]

"Podría decirte que eh:: normal que me molestan (...) cuando yo vivía en Calama ahí co::mo que no me molestaban después llegue a Temuco como que me molestaban por el apellido cosa que acá en Temuco no debería ser as'́".

[líneas 167 a 169 ]

(...) "en cambio como que yo soy así soy como más como más de la ciudad [risa] entonces no me siento tanto del campo tan mapuche porque la mayoría de la gente que son mapuche son del campo no la mayoría, pero no todos" T:M:Cla.

Lo anterior nos lleva a hipotetizar que las cualidades negativas atribuidas a la ciudad son discursivamente mitigadas mediante la estrategia de protección de imagen (face) frente al interlocutor, como asimismo debido a las ventajas que la ciudad ofrece al joven en términos de su desarrollo personal y profesional. Cabe señalar que sobre el $80 \%$ de los jóvenes entrevistados, principalmente de Santiago y en menor número de Temuco, plantearon que no se irían a vivir al campo con sus comunidades, sino que prefieren mantener el vínculo con ellas por intermedio de visitas y estadías cortas.

\section{Espacios culturales recreados}

Los espacios recreados surgen producto de la migración desde las áreas originarias del pueblo mapuche, principalmente región de La Araucanía y del Biobio, hacia las ciudades. Una vez que el proceso de migración ha sido llevado a cabo y al no encontrar espacios en los nuevos lugares de residencia que representen y refuercen aspectos de su cultura, los adolescentes (en la mayoría junto a sus padres) buscan generar espacios en sus comunas y barrios los que recrean para representar y simbolizar los espacios originales ancestrales. Ello se observa en el relato de $\mathrm{V}$ de Santiago respecto de una cancha otorgada por la Municipalidad de La Pintana donde hay construida una $r u k a^{3}$ y espacio para hacer ngillatun ${ }^{4}$ y jugar palin $^{5}$ :

"Entrevistador: ¿(...) y tú participas siempre?

V.: Sí pero de repente no más cuando aquí hacen algo venimos, pero no casi siempre... hacen ngillatún o a veces jugamos palín o no especialmente aquí en otro lugar igual voy con mi papá a jugar" S:H:V.

\footnotetext{
${ }^{3}$ La ruka es el nombre de la vivienda tradicional de los mapuches, construida en forma hexagonal, de paja y madera con un espacio redondo en su cielo para permitir la salida del humo del fogón central.

${ }^{4}$ El ngillatun es la ceremonia más importante del pueblo mapuche de agradecimiento y rogativa al Dios Ngenechen.

${ }^{5}$ El palín es el tradicional juego mapuche, similar al juego de hockey, pero que se juega con palo y pelota de madera.
} 
El joven $\mathrm{V}$ se posiciona como miembro participante del espacio recreado en donde se llevan a cabo actividades culturales mapuches. El rol del espacio recreado para este adolescente es establecer una relación con actividades típicas de la cultura mapuche que practica junto a su padre. Se observa en su discurso la estrategia de apelación a la autoridad, el padre, lo que indica que se trata de una persona importante en la relación del adolescente que lo vincula con actividades de la cultura. El relato de $\mathrm{V}$ muestra también que no solo es necesario encontrar y acomodar un espacio que recree características del espacio original, sino que ello se hace con el propósito de establecer allí un cierto tipo de interacción familiar, social y cultural. Al mismo tiempo, el joven plantea la flexibilidad de los espacios donde se recrea la cultura, por cuanto no sólo se practican en el lugar más cercano a donde se habita, sino que además pueden llevarse a cabo en otros lugares.

Para algunos jóvenes de Temuco los espacios recreados en la ciudad no forman parte de las prácticas culturales mapuches auténticas, de modo que no las reconocen como tal. En el relato de $\mathrm{C}$ se puede observar nuevamente el dilema entre "ser" y "sentirse" mapuche: se "es" mapuche en la ciudad, pero uno se "siente" mapuche en el campo donde se practican las tradiciones mapuches en sus espacios típicos.

"Entrevistador: (...) pero por ejemplo en actividades culturales, no participan tu familia o tú, a un we tripantu, por ejemplo

C.: en general, no, como que::, > es que como que somos de la ciudad< no / no practicamos eso, porque a mi papá no le enseñaron así, eso se hace en el campo, ahí se siente uno como más mapuche". T:M:C.

El relato anterior deja en evidencia que las actividades típicas de la cultura mapuche como el ngillatún no son parte del entorno urbano, sino que se asocian directamente al "campo". Más aún, el entrevistado enfatiza el hecho que la ciudad es un espacio completamente diferente dónde éstas actividades no se llevan a cabo "es que como que somos de la ciudad< no / no practicamos eso". Esta última frase constituye una afirmación enfática de la no correspondencia entre urbanidad y tradiciones mapuches.

Una discusión aparte merece el rol atenuador del marcador discursivo "como/como que" de alta frecuencia en el discurso de los jóvenes entrevistados (Oteíza y Merino, 2012). Se trata de un recurso modalizador que puede expresar una expresión de duda o inseguridad frente a un argumento complejo o dilemático que el entrevistado está construyendo, que va asociado también a la protección de la imagen del hablante frente a su interlocutor, y por lo tanto el entrevistado se "toma su tiempo" para seleccionar las frases y palabras adecuadas de su repertorio discursivo. Ello puede observarse, por ejemplo, cuando C más arriba se posiciona respecto de no participar en actividades culturales en la ciudad explicita su duda 
sobre cómo enfrentar el dilema de categorizarse étnicamente "como que::,>es que como que somos de la ciudad...", o bien cuando Cla planteaba más arriba su dilema de reconocerse y sentirse mapuche en la ciudad de Temuco donde ha percibido instancias de discriminación en su contra "en cambio como que yo soy así, soy como más como más de la ciudad". No obstante en otros casos, el marcador "como/como que2 funciona como despersonalización o distanciamiento del objeto del discurso. Regularmente este marcador va junto a construcciones sintácticas de tercera persona.

\title{
3. La escuela y el liceo
}

Otro espacio urbano donde los adolescentes entrevistados recrean prácticas culturales mapuches es la escuela y en bastante menor grado el liceo. Los adolescentes se identifican con estos espacios cuando en ellos se abren espacios para la práctica de actividades culturales, por ejemplo, la celebración de wetripantu o año nuevo mapuche, como se observa en el relato de $\mathrm{J}$ de Temuco. Entrevistador: "y aquí se respeta la cultura mapuche?".

\begin{abstract}
"En este colegio sí. Creo que esa es una de las cosas que me gusta de este colegio, porque aquí celebramos el wetripantu, entonces se hacen... yo me vestí pa'l wetripantu de mapuche y todos se reían porque se veía raro que yo me vistiera. Pero me gusta eso porque como mi familia lo celebra aquí puedo representar mi raza y eso me gusta". T:M:J.
\end{abstract}

Como se aprecia en el extracto, el adolescente manifiesta su identificación con el espacio escuela debido a que allí se celebran regularmente actividades culturales mapuches, de modo que $J$ no se siente discriminado por sus compañeros cuando recuerda que "todos se reían porque se veía raro que yo me vistiera (de mapuche)", sino que lo interpreta como un evento positivo, novedoso para sus compañeros por la vestimenta que llevaba y como un espacio donde puede representar a su cultura.

Los adolescentes de ambas ciudades identifican y valoran las características positivas de los espacios urbanos y las ventajas que estos ofrecen en cuanto al desarrollo académico y económico, y son los jóvenes de Santiago quienes principalmente se refieren a los espacios recreados facilitados por entidades gubernamentales, juntas de vecinos y municipalidades, los cuales al parecer son más asequibles en términos de lejanía con el territorio ancestral mapuche en el sur del país. Por otro lado, los jóvenes de Temuco se refieren a los espacios recreados como estimuladores de identidad étnica tomando como ejemplo central la escuela, ya que, debido a la cercanía geográfica de Temuco con las comunidades mapuches, la escuela se constituiría en la instancia que mejor recoge la necesidad de los niños y jóvenes mapuches de ser valorados en su 
identidad étnica. Ello podría indicar que la naturaleza de los espacios recreados para los adolescentes puede ser diferente dependiendo de las condiciones de cercanía/lejanía de los lugares de origen ancestral; sin embargo el rol de movilizador de identidad étnica que se le atribuyen a estos no presenta variación.

\section{CONCLUSIONES}

Coincidimos con Merino y Tileaga (2010) que los adolescentes recrean espacios que representen los lugares originales de la cultura en un intento por preservar la unicidad propia del pueblo mapuche y con el fin de establecer relaciones interpersonales semejantes a las originarias de la cultura. Estas actividades y los lugares en los que se realizan son parte de la unicidad de los miembros de la cultura mapuche, y constituyen elementos diferenciadores y marcadores de su propia identidad étnica.

Los procesos migratorios de los miembros de las comunidades mapuches del campo a la ciudad se justifican esencialmente debido a las oportunidades de trabajo, económicas y también académicas que el entorno urbano ofrece. $\mathrm{Al}$ ocurrir este fenómeno, los adolescentes mapuches buscan y recrean entornos que representen aquellos que son parte de su cultura y que permitan "sentirse en casa" (Dixon y Durrheim, 2000). En estos lugares se celebran actividades características de la cultura mapuche, ya sea de forma permanente o no permanente como es el caso de las escuelas, principalmente en Temuco.

Lo anterior demuestra que los espacios culturales se recrean en un contexto urbano debido a la participación de los tres procesos cognitivos planteados por Proshansky et al. (1983). El acondicionamiento de un espacio representativo de la cultura propia permite satisfacer las necesidades identitarias de las personas, es decir, el acomodo de los espacios para establecer un entorno similar al original. Luego se produce la recreación de interacciones sociales en los espacios adaptados donde los adolescentes buscan recrear tipos de interacciones culturales que permitan celebrar y participar en actividades típicas mapuches. Finalmente, la modificación de la identidad en términos de adaptar la propia identidad al espacio recreado teniendo en consideración que este no es idéntico al original, de modo que los jóvenes deben "negociar su interculturalidad" (Merino, 2012). Ello significa "traer" simbólicamente un lugar rural a un entorno urbano el que, si bien no es una réplica exacta del espacio original, el nuevo no afecta la percepción que los adolescentes poseen del lugar originario. Ello muestra que la identidad se constituye para estos jóvenes en un constructo cambiante que evoluciona y se adapta a diferentes contextos y situaciones.

En consecuencia, el rol de los espacios recreados es reforzar la construcción de identidad étnica de los adolescentes que desean identificarse o reforzar su etnicidad; es decir, los adolescentes que se identifican como mapuches, ya sea de forma activa o pasiva, valoran la recreación de espacios, ya 
que es en ellos donde aprenden a conocer la cultura y las prácticas tradicionales. No obstante, en la mayoría de los adolescentes mapuches urbanos la identificación con la cultura y con los espacios asociados tradicionalmente a la cultura mapuche es dilemática lo que se observa en el discurso de los jóvenes mediante la disyuntiva moral entre "ser" y "sentirse" mapuche y el despliegue de atenuadores discursivos ("como/como que"). Ello muestra la presencia de modalidades retórico-discursivas de protección de la propia imagen en un entorno de relaciones interétnicas e interculturales asimétricas entre la sociedad chilena y el pueblo mapuche.

Universidad Católica de Temuco*

Facultad de Educación

Rudecindo Ortega 02950, Temuco (CHILE)

hturra@uctemuco.cl

OBRAS CITADAS

Aillahuil, Néstor. "Efectos de la presencia de la escuela en la identidad mapuche, en niños que asisten a la escuela de Roble Huacho". Temuco: Universidad Católica de Temuco, 1996.

Altman, Irwin, and Setha Low. "Place Attachment". Vol. 12. New York: Springer, 1992.

Aravena, Andrea. "Los Mapuche-Warriache: Procesos migratorios contemporáneos e identidad mapuche urbana", en Revista América Indigena 54.4 (2003):162-88.

Barros, Claudia. "Identidades entre lo urbano y lo rural". Universidad de São Paulo. São Paulo. 20 Mar. 2005. Rescatado el 5 Feb. 2012. Disponible en http://www.observatoriogeograficoamericalatina.org.mx/egal10/Geografi asocioeconomica/Geografiacultural/07.pdf.

Bello, Álvaro. "Migración, identidad y comunidad mapuche en Chile. Entre utopismos y realidades". Asuntos Indígenas 3 Abr. 2002: 40-48.

Benwell, Bethan and Elizabeth Stokoe. "Discourse and Identity". Vol. 1. Edinburgh: Edinburgh University Press, 2006. 
Héctor Turra, Sandra Garrido, Chery Pérez, Gabriel Llanquinao, María Eugenia Merino

Castells, Manuel. "La cuestión Urbana". 16 ed. México: Siglo XXI editores, 2004.

Corbett, Michael. "Rural Education and Out-Migration: The Case of a Coastal Community". Canadian Journal of Education 28.1-2 (2005): 52-72.

Corbett, Michael. "Travels in space and place: Identity and rural schooling". Canadian Journal of Education 30.3 (2007): 771-92.

Danziger, Kurt. "Naming the Mind: How Psychology Found Its Language". Vol. 1. Londres: Sage Publications Ltd, 1997.

Dixon, John, and Kevin Durrheim. "Dislocating identity: Desegregation and the transformation of place", en Journal of Environmental Psychology 24.4 2004:455-473.

------- "Displacing place-identity: A discursive approach to locating self and other". British Journal of Social Psychology 39.1 Mar. (2000): 27-44.

Edwards, Derek. "La psicología discursiva", en Análisis del Discurso: Manual para las Ciencias Sociales. Barcelona: Edicions de la Universitat Oberta de Catalunya, 2003:141-156.

------ "Discourse and Cognition". Londres: Sage Publications Ltd, 1997.

Edwards, Derek y Jonathan Potter. "Discursive Social Psychology", en Handbook of Language and Social Psychology. West Sussex: Wiley, 2000.

"Discursive Psychology". London: Sage 1998.

Hall, Stuart. "Foucault: Power, Knowledge and Discourse". Discourse theory and practice: a reader. Ed. Margaret Wetherell, Simon Yates, and Stephanie Taylor. London: Sage Publications Ltd, 2001.

Hauge, Ashild. "Identity and place: a critical comparison of three identity theories". Architectural Science Review 43.31 Mar. (2007): 44-55. Rescatado el 6 Ene. 2012. Disponible en:

http://faculty.arch.utah.edu/benham/group\%203/Place-Identity.pdf.

Heimstra, Norman, y Leslie McFarling. Psicología Ambiental. México: El manual moderno, 1976.

Hepburn, Alexia y Sandra Wiggins. "Size matters: constructing accountable bodies in NSPCC helpline interaction". Discourse and Society,16, 62546. 2007.

Hidalgo, Carmen. Apego al lugar: ámbitos, dimensiones y estilos. Tenerife: Universidad de la Laguna, 1998.

Hormuth, Stephan. The Ecology of the Self Relocation and Self-Concept Change. Cambridge: Cambridge University Press, 2010.

Knez, Igor. "Attachment and identity as related to a place and its perceived climate." Journal of Environmental Psychology 25.2 (2005):207-18.

Kropff, Laura. "Mapurbe': jóvenes mapuche urbanos", en Kairós Revista de Temas Sociales 14 Oct. (2004). Rescatado el 6 Ene. 2012. Disponible en: http://www.revistakairos.org/k14-archivos/laura\%20kropff.pdf. 
Lalli, Marco. "Urban Identity.” Environmental social psychology. Ed. David Canter. Dordrech: Kluwer Academic Publisher, 1988:303-11.

McAndrew, Francis. "The measurement of 'rootedness' and the prediction of attachment to home-towns in college students". Journal of Environmental Psychology 18.4 (1998):409-15.

Merino, María E. David Mellor, José L. Saiz, and Daniel Quilaqueo. "Perceived discrimination amongst the indigenous Mapuche people in Chile: some comparisons with Australia". Ethnic and Racial Studies 32.5 (2009): 80222. Impreso.

Merino, María E., and Christian Tileaga. "The construction of ethnic minority identity. A Discursive psychological approach to ethnic self-definition in action". Discourse and Society 22.1 (2010):86-101. Web. 8 Ene. 2010.

Merino, María E. "The impact of educational settings on the construction of ethnic identity among urban mapuche adolescents in Chile" en Rosa Bruno-Jofre y James Scott Johnson (eds) Teacher Education in a Transnational World. University of Toronto Press, 2012. Incorporar Ciudad.

Oteíza, Teresa y Merino, María Eugenia. "Am I genuine mapuche? Tensions and contradictions in the construction of ethnic identity in Mapuche adolescents from Temuco and Santiago". Discourse \& Society. 23(3) 121. (2012).

Papastergiadis, Nikos. The turbulence of migration: globalization, deterritorialization, and hybridity. Cambridge: Polity Press, 2000.

Proshansky, Harold. "The City and Self-Identity". Environment and Behavior 10.2 Junio (1978):147-69.

Proshansky, Harold, Abbe Fabian, and Robert Kaminoff. "Place-identity: Physical world socialization of the self". Journal of Environmental Psychology 3.1 (1983):57-83.

Tajfel, Henri y Turner, John. "An Integrative Theory of Inergroup Conflict", en The Social Psychology of Intergroup Relations. Monterey, CA: BrooksCole, 1979.

Tripailaf, Daniel. "Discriminación racial y social crea complejo de inferioridad en el pueblo mapuche". Revista Cauce 46 (1969):4-8.

Twigger-Ross, Clare, and David Uzzel. "Place and identity processes." Journal of Environmental Psychology 16 (1996): 205-22. Web. 2 Ene. 2012.

Valera, Sergi. "Análisis de los aspectos simbólicos del espacio urbano. Perspectivas desde la psicología ambiental", en Revista de Psicología Universitas Tarraconensis 18.1 (1996):63-84.

Valera, Sergi, and Enric Pol. "El concepto de identidad social urbana: una aproximación entre la Psicología Social y la Psicología Ambiental”, en Anuario de Psicología 62.3 (1994):5-24. 
Héctor Turra, Sandra Garrido, Chery Pérez, Gabriel Llanquinao, María Eugenia Merino

Wetherell, Margaret. Theorizing Identities and Social Action. Palgrave Macmillan, 2009.

Wetherell, Margaret and Jonathan Potter. Mapping the Language of Racism: Discourse and the Legitimation of Exploitation. Londres: Harvester/Wheatsheaf, 1992. 